\title{
Visual Analysis of Learning Evaluation Research Under the B-Learning
}

\author{
Duan Fang, Li Hong \\ Information Institute, Yunnan Normal University, Kunming, China
}

Email address:

1595003630@qq.com (Duan Fang)

\section{To cite this article:}

Duan Fang, Li Hong. Visual Analysis of Learning Evaluation Research Under the B-Learning. Science Innovation. Vol. 8, No. 4, 2020, pp. 97-101. doi: 10.11648/j.si.20200804.13

Received: May 30, 2020; Accepted: July 14, 2020; Published: July 23, 2020

\begin{abstract}
It provides important reference for the researchers of learning evaluation under the B-Learning. This paper takes the subject literature of learning evaluation under the B-Learning published in CNKI database from 2010 to 2020 as the research object, uses the literature analysis method and visual analysis method, combs the subject and key words of relevant domestic literature with the help of CiteSpace software, and evaluates the learning under the B-Learning in the past ten years The key nodes and clustering are analyzed. It is found that the research on this subject mainly focuses on three categories: evaluation tools, evaluation methods and applications; the research focuses on four aspects: evaluation methods, evaluation standards, evaluation models and evaluation tools; and the trend towards the strengthening of information-based evaluation tools. Based on the above analysis and conclusion, in the future, the following three points should be paid attention to in the study of learning evaluation under the B-Learning in China: making effective use of big data; strengthening the construction of the integrated intelligent learning evaluation system; building a standardized and easy to operate evaluation index system.
\end{abstract}

Keywords: B-Learning, Learning Evaluation, Visual Analysis

\section{混合式教学模式下学习评价研究的可视化分析}

段芳, 李红

云南师范大学信息学院, 昆明, 中国

邮箱

1595003630@qq.com（段芳）

摘要: 为混合式教学模式下学习评价研究者提供重要参考, 本文以CNKI数据库中2010-2020年发表的混合式教学模式 下学习评价主题文献作为研究对象, 采用文献分析法和可视化分析法, 借助Citespace软件梳理了国内相关文献的主题 和关键词, 对近十年混合式教学模式下学习评价研究的关键节点和聚类进行分析。研究发现, 该主题的研究主要围绕 评价工具、评价方法及应用三个大类进行开展研究; 研究热点主要集中在评价方式、评价标准、评价模型、评价工具 四个方面, 并向着信息化的评价工具不断加强的趋势。基于以上分析与结论, 在未来, 国内有关混合式教学模式下学 习评价研究应关注以下三点: 有效利用大数据; 加强一体化智能学习评价系统建设; 构建规范、易操作的评价指标体 系。

关键词: 混合式教学模式, 学习评价, 可视化分析 


\section{1. 前言}

《国家中长期教育改革和发展规划纲要》指出, 信息 技术对教育发展具有革命性影响, 要加快教育信息化进程, 加强优质教育资源开发与应用, 开发网络学习课程, 建立 灵活开放的资源公共平台, 促进优质教育资源普及与共享。 改革教育评价和人才评价制度, 根据培养目标和人才理念, 建立科学多样的评价指标, 探索促进学生发展的多种评价 方式, 激励学生乐观向上, 独立自主[1]。在国家中长期教 育改革推动下, 混合式教学呈现出喷井式发展。混合式教 学模式是指利用信息技术将传统课堂的面授教学与在线 学习有机结合，从而提高学习效果 [2]。随着“互联网+”时 代的到来, 冯晓英等人认为混合式教学是基于移动通讯设 备、网路学习环境与课堂讨论相结合的教学情景, 强调为 学生创造一种高度参与、个性化学习情景[3], 充分体现教 师主导学生主体的教学理念。学习评价是混合式教学模式 中的重要环节, 高度参与、个性化学习情景的创设离不开 有效的教学评价。

\section{2. 研究设计}

本文主要聚焦于国内混合式教学模式下学习评价的 研究热点、研究前沿和关键词聚类这三个方面。借助 Citespace软件, 通过内容分析法对检索到的文献进行分析, 绘制了混合式教学模式下学习评价的知识图谱, 从而对混 合式教学模式下学习评价的研究现状进行梳理与阐述。

\section{1. 数据来源}

本文以 CNKI数据库为数据来源, 以“混合式”和“学习 评价”为主题词进行检索，选定文献发表时间为
2010-2020年，剔除无关键词、与主题相关度不高的无效 数据, 得到有效文献304篇。

\section{2. 研究方法}

本研究先对所笁选的文献数据 $\mathrm{xt}$ 文档进行预处理, 再 导入到Citespace软件中进行知识图谱的生成。Citespace具 体参数设置如下：时间段为 $2010-2020$ 年, 时间切片为 1 年，节点类型为“主题 (Title) ”和“关键词 (Keyword)”, 阈值赋值 ( Thresholds) 中 $\mathrm{c}, \mathrm{cc}, \mathrm{cc}$ 的值为: $0,6,30 ; 2,6,30 ; 4,6,25$, 呈现相关可视化图谱。

\section{3. 研究结果}

\section{1. 关键节点分析}

\subsection{1. 研究热点}

关键词用来表达文献的主题内容, 是研究者学术思想、 观点的凝练, 从一定程度上能反映出研究的核心。因此, 关键词出现的频率与研究热度正相关。以样本数据中的主 题词和关键词为研究依据, 绘制出高频关键词和关键词的 可视化图谱, 可以确定混合式教学模式下学习评价的研究 热点。

关键词可视化图谱如图 1 所呈现的网络节点个数 $\mathrm{N}=42$, 节点代表关键词, 字体的大小代表关键词出现频 次的多少; 连线数量 $\mathrm{E}=68$, 节点间的连线反映了关键词之 间的共现情况, 线条粗细表示共现强度; 网络密度 Density $=0.079$ ，表示节点间联系的紧密度; “十”字表示关 键词的中心度性, “十”字的大小与中心性的强弱和影响力 的大小成正比。

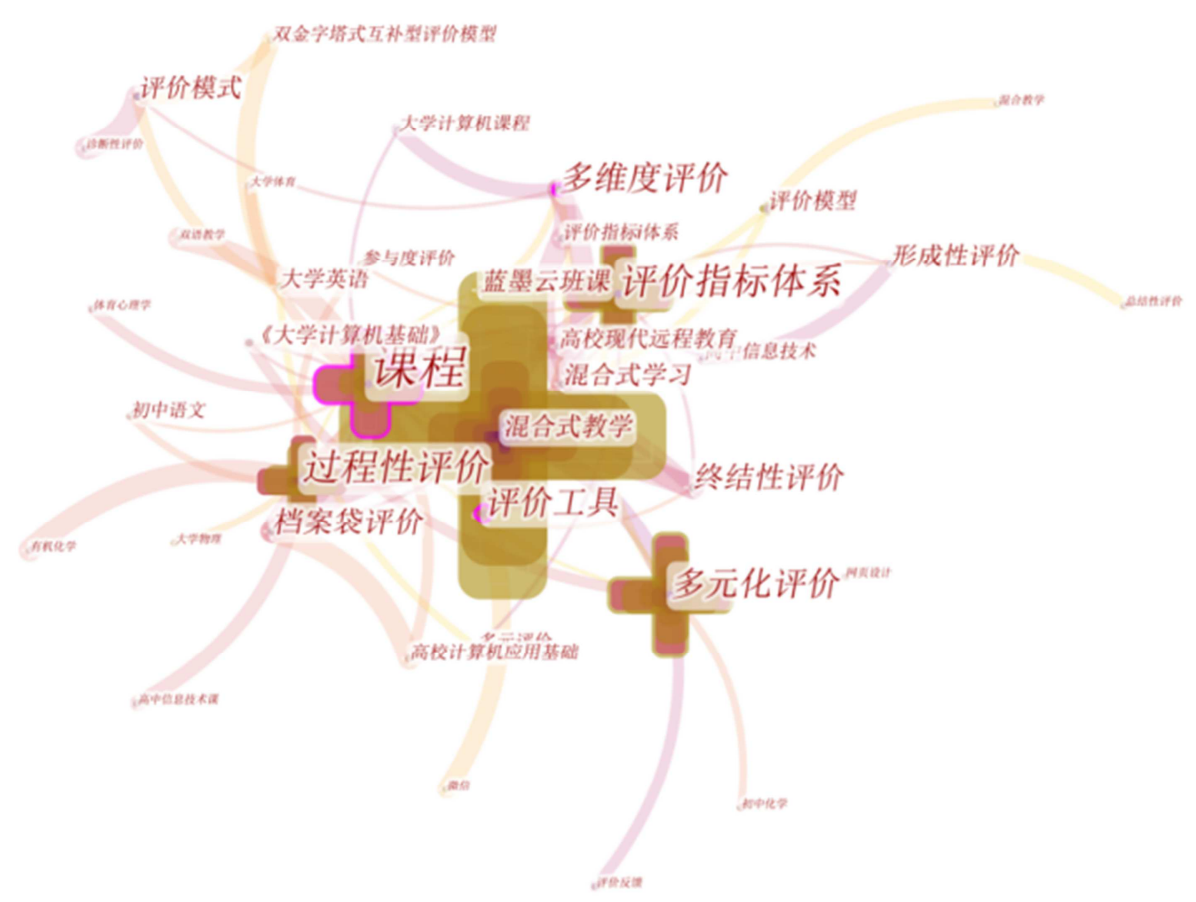

图1 关键词可视化图谱。 
表1 高频关键词。

\begin{tabular}{llllllll}
\hline 关键词 & 频次 & 中心度 & 出现年份 & 关键词 & 频次 & 中心度 & 出现年份 \\
\hline 混合式教学 & 272 & 0.05 & 2010 & 评价模型 & 16 & 0.10 & 2012 \\
多元化评价 & 128 & 0.21 & 2010 & 形成性评价 & 14 & 0.15 & 2012 \\
评价指标体系 & 105 & 0.36 & 2010 & 混合式学习 & 13 & 0.09 & 2012 \\
课程 & 88 & 0.65 & 2011 & 终结性评价 & 11 & 0.03 & 2012 \\
过程性评价 & 81 & 0.26 & 2012 & 评价模式 & 9 & 0.18 & 2010 \\
评价工具 & 20 & 0.32 & 2013 & 蓝墨云班课 & 8 & 0.14 & 2018 \\
多维度评价 & 18 & 0.38 & 2011 & 大学英语 & 6 & 0.04 & 2017 \\
\hline
\end{tabular}

根据普赖斯定律, 确定 $>=6$ 的关键词为高频词, 本研 究通过对高频词进行综合分析而判定热点。高频关键词如 表1所示, 表 1 显示共有 14 个热点关键词, 除去基本关键词 “混合式教学”、“混合式学习”后得到12个热点关键词。12 个热点关键词大致可归纳为五个层面。(1)评价方式层面的 高频词有：多元化评价、多过程性评价、评价、形成性评 价、终结性评价, 从五种评价方式的频次来看, 多元化评 价、过程性评价、多维度评价得到越来越广泛的认可, 说 明单一的评价方式已无法满足混合式教学模式下人才培 养的需求; (2)评价标准层面, 主要涉及高频词“评价指标 体系”, 通过建立评价指标体系使评价结果更加科学;(3)评 价模型层面, 主要涉及高频词“评价模型”、“评价模式”, 评价模型的构建使评价更系统, 更具操作性; (4)工具层面, 主要涉及高频词“评价工具”、“蓝墨云班课”, 随着信息技
术的发展, 信息化评价工具也随之成为研究的热点, “蓝 墨云班课”作为当前主流的教学评价辅助工具, 可促进评 价的客观性和科学性, 从而提升学生的学习效果; (5)应用 层面, 主要涉及高频词“课程”、“大学英语”, 通过分析课 程特征, 提出有效的评价方案, 建立评价标准, 促进更好 的评价, “大学英语”是开展混合式教学的主要学科, 也是 混合式教学模式下学习评价研究的热门学科。

\subsection{2. 研究前沿}

为了从时间维度上显示前沿关键词的演进, 本研究采 用Citespace中“时区视图”的呈现方式来展现关键词的演 进, 如图2所示。

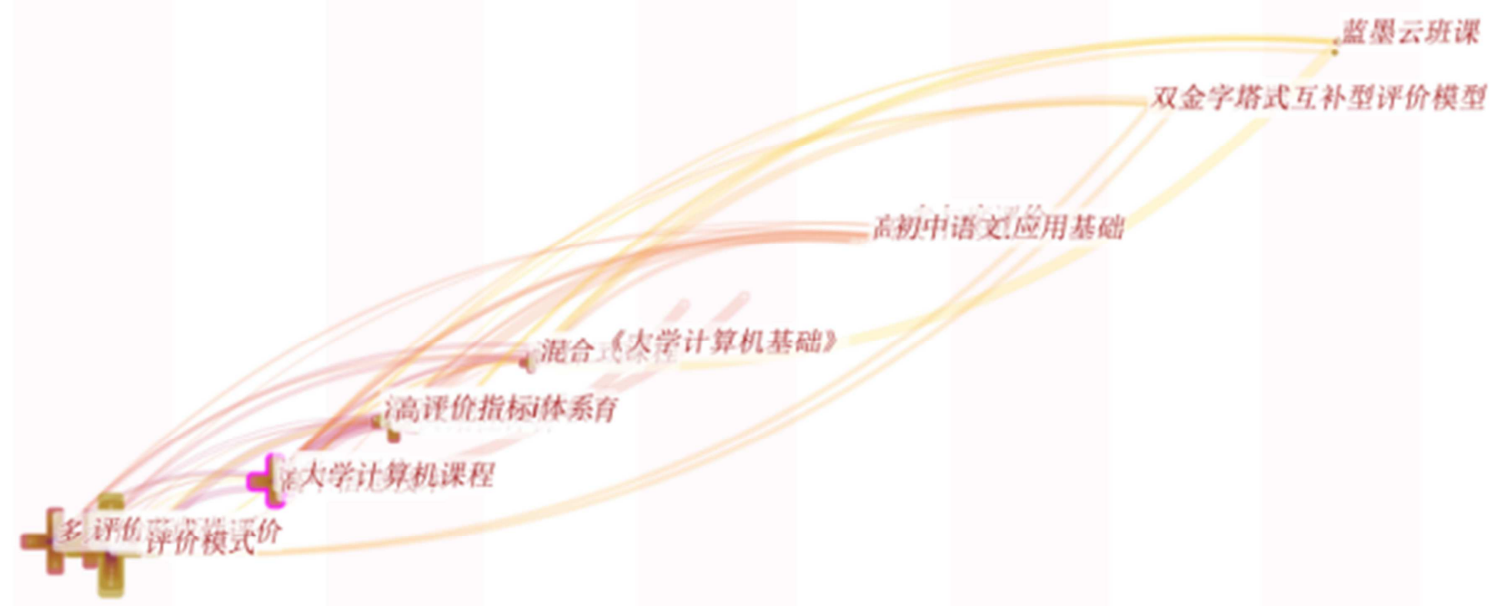

图2 时区视图。

从关键词的演进来看, 近十年的研究的趋势逐渐从评 价方式、评价模式过渡到课程应用再到信息化评价工具研 究。蓝墨云班课是当前混合式教学模式下被广泛应用的评 价系统, 教师可通过手机开展一系列教学活动, 如向学生 发布公告、共享资源、发布作业、批改作业、开展头脑风
暴、组织讨论、在线答疑等, 同时还支持教师组织小组学 习及同伴互评活动, 关键在于能有效追踪学生的学习轨迹, 记录学生的学习情况, 通过 “经验值” 来衡量学生的学习程 度 [4]。蓝墨云班课作为一款专门为混合式教学环境下教师 
开展教学活动而打造的教学工具, 为该模式下的学习评价 提供巨大的应用价值。

\section{2. 聚类分析}

本研究使用对数似然率算法 ( Log Linkelihood Rate,LLR）对关键词网络进行聚类, 得到的结果如图3所 示, Modularity $\mathrm{Q}=0.4987(>0.3)$ 说明网络社团机构较为 显著; Mean Silhouette $=0.5111$ ( $>0.5$ ) 说明聚类结果合理, 反映出了混合式教学模式下学习评价研究主题相对集中、 明确。由图 3 可知, 共得到 6 个聚类, 可将这 6 个聚类归纳 为三个部分：评价工具（\#0评价工具）、评价方法（\#2 多元化评价、 $\# 3$ 过程性评价、 $\# 5$ 形成性评价) 、应用（\#1 课程、\#4大学英语）。

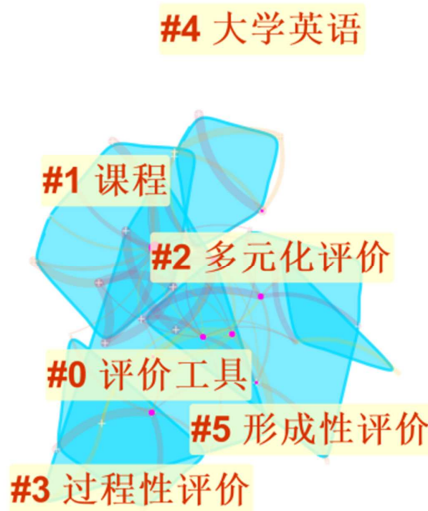

图3 关键词聚类。

\subsection{1. 日益注重信息化评价工具的研究与应用}

所谓评价工具包含两个方面, 其一为评价量表即评价 指标, 是评价结果公正、科学的依据, 贯穿混合式教学模 式下的各个环节; 其二为信息化的评价平台, 随着混合式 教学模式的不断演进和完善, 信息化评价平台也逐渐得到 发展。目前市场上有智慧树、学习通、网易公开课等多套 商业化基于 Web的支持学习评价的学习系统, 同时随着信 息技术的创新, 基于移动终端的教学助手也逐渐被用于混 合式教学活动中, 如雨课堂、微助教、蓝墨云班课等[5]。

\subsection{2. 评价方式更加注重多元化和过程性}

多元化评价通过协调各评价主体, 运用多样化的评价 方式, 对学生的行为变化进行全面化的评价, 以此促进学 生个性化发展, 提高科学素养[6]。即, 评价方式多元化[7]: 改变传统以目标结果为导向的终结性评价, 将诊断性评价、 形成性评价和终结性评价进行有机融合, 对学生的学习进 行有效融合; 评价主体多元化 [8]: 采用“教评+自评+互评” 的方式, 保证评价的客观性; 评价对象多元化: 从学生的 知识、技能、情感、态度等多个维度开展评价, 促进学生 的全面发展。

过程性评价将评价与学生学习过程紧密结合, 从关注 教育结果转向关注教育过程, 强调知识构建过程中的反馈 与及时指导, 关注师生间的交流与互动 [9]。混合式教学模 式下, 通过分析学生线上线下的学习行为表现, 对学生整 个学习过程进行综合评价, 为学生提供反馈, 有助于学生
发现学习过程中的不足, 达到最佳的学习效果, 同时能够 促进教师对教学环节进行反思, 提高教学效果。

\subsection{3. 注重实践研究}

国内混合式教学正处于喷井式发展阶段, 已融入各高 校、职业院校课堂中, 在中小学课堂中也逐渐被引入, 对 教学改革有较强的促进作用 [10]。随着混合式教学模式的 推广, 研究者们基于不同学科课程背景进行学习评价的实 证研究, 为课程评价研究提供了参考。随着经济全球化的 推进, 社会对人才的综合素质提出了越来越高的要求, 从 而语言类的学习备受关注, 其中最为突出的是大学英语课 程。语言学习注重学习环境与个性化, 互联网的普及为学 生的语言学习提供了超越时空限制、形象、多彩的学习环 境。随之带来评价方式的改变, 对于“新环境”下英语学习 评价从传统的“哑巴英语”、“中国式英语”转化为注重学生 的英语交际能力, 采用多维度多元化的评价方式, 对学生 进行综合评价[11]。

\section{4. 思考}

近十年, 国内混合式教学模式下学习评价得到了不断 的发展和完善, 笔者认为在未来发展中应关注以下几点:

\section{1. 有效利用大数据}

在信息化的时代背景下, 大数据在各行各业中发挥出 了无限的潜力。混合式教学模式下, 过程性评价是个性化 自主学习过程的重要组成部分, 教育大数据技术为实现混 合式学习的过程性评价提供了可能[12]。教师应有效利用 学生线上线下产生的学习行为数据对学生整个学习过程 进行动态评价, 随时时追踪了解学生学习动态, 基于数据 驱动, 促进个性化和精准化教学[13]。

\section{2. 加强一体化智能学习评价系统建设}

传统的测试无法检测学生的学习素养, 基于线上线下 学习数据一体化学习反馈智能化的评价系统是当前的诉 求。

线上线下行为数据一体化: 线上学生行为数据容易记 录, 但线下课堂教学过程中的学习数据依赖于教师纯手工 记录, 给教师增添了负担, 同时会因为各种客观原因导致 评价结果不准确。因此, 本着操作方便、减轻教师负担同 时又不影响教师正常教学秩序的原则下, 需要在线上教学 系统基础上增加线下学习行为数据记录分析程序, 促进评 价一体化。

反馈智能化即：反馈的及时性与双向性[14]。通过分 析学生行为数据, 教师和学生双方能够及时收到反馈信息, 促进学生自我认知、自主学习, 教师动态调整教学过程, 以满足个性化教学需求。在双向反馈的过程中, 不断强化 师生双方的交流与互动。

\section{3. 构建规范、易操作的评价指标体系}

当前不乏对混合式教学模式下学习评价指标体系构 建研究, 但限于指标涉及各个科目课程, 比较分散、实施 
复杂、不易迁移, 因此需要结合混合式教学模式的特点和 不同层次人才培养方案，综合各科课程特征，建立基于不 同层次的、统一、规范、易操作、易迁移的混合式教学模 式下学习评价指标体系。

\section{5. 结语}

随着信息技术的不断深化和发展, 教育逐渐由信息化 走向智慧化 [15], 过程性评价系统如何支持混合教学模式 下课程智慧化发展将成为研究者们广泛关注的问题 [16][17], 其研究空间也非常大。本文通过梳理CNKI数据 库中近十年的相关文献, 分析了混合式教学模式下学习评 价的研究现状、热点及研究前沿, 同时给出合理化的建议, 为混合式教学模式下学习评价研究者提供一定的参考价 值。

\section{参考文献}

[1] 中共中央国务院印发国家中长期教育改革和发展规划纲 要．中华人民共和国国史网 [引用日期2016-07-25]。

[2] 刘婷,陈瑶.慕课支持下的混合式教学模式实验研究一以 “实用日语（上）”慕课为例 [J]. 北京: 现代教育技 术, $2019,(12): 55-60+40$.

[3] 冯晓英,王瑞雪, 吴怡君, 等. 国内外混合式教学研究现状述评 一基于混合式教学的分析框架 [J]. 北京:远程教育杂 志,2018,(3):13-24.

[4] 赵冤,姚海荣. 基于蓝墨云班课的混合式教学行为研究以 “现代教育技术”课程为例 [J]. 北京: 现代教育技 术, 2019,(5):46-52.
[5] 谭志虎, 胡迪青,田媛媛, 许炜. 微助教对高校大班课堂互动教 学的重构[J].北京:现代教育技术,2018,(1):107-113.

[6] 廖继昌. 多元化教育评价初探 [EB/OL].http://blog.cersp.com/18886/672081.aspx,2006-09-1 7.

[7] 熊才平,戴红斌,葛军.教育技术: 研究进展及反思 [J].教育研 究,2018,(3):118-128.

[8] 郭学品, 黄成, 周玉萍.混合式教学质量评价模型分析[J].湖北: 湖北函授大学学报,2018,(7):129-131.

[9] 韩叶秀,杨成. 认识论视野下学习过程性评价探析 [J]. 北京:教 育探索,2012,(2):5-7.

[10] 杨歌谣.混合式教学模式下教学评价的文献综述与展望 $[\mathrm{J}]$. 高教论坛,2019,(2):64-67.

[11] 刘洋.混合式大学英语教学模式实证研究 以《英语 国家概况》英语课为例 $[\mathrm{J}]$. 湖北: 湖北经济学院学 报,2019,(9):153-155.

[12] 上超望,韩梦,刘清堂.大数据背景下在线学习过程性评价系 统设计研究[J].中国电化教育,2018,(5):90-95.

[13] 李振超,陈琳,郑旭东.大数据理念下的发展性学习评价系统 设计研究[J].现代教育技术,2015,(6):108-114。

[14] 韩叶秀,杨成.认识论视野下学习过程性评价探析[J].教育探 索,2012,(2):5-7.

[15] 陈琳,陈耀华,乔灿,等.教育领域综合改革开局之年我国教育 信息化新发展[J].中国电化教育,2015,(1):138-145.

[16] 陈琳,陈耀华,郑旭东,等.智慧教育 中国引领[J].电化教育研 究,2015,(4):24-29.

[17] 陈琳,王运武.面向智慧教育的微课设计研究 [J].教育研 究,2015,(3):127-130. 